\title{
Estudio de la reoxidación y carburización de pelets reducidos de mineral de hierro ${ }^{(\bullet)}$
}

\author{
J. Lemus R. ${ }^{(*)}$, G. Mendoza S. ${ }^{(* *)}$ y E. Bedolla B. ${ }^{(* *)}$ \\ Resumen Se estudió la influencia de la carburización en la reoxidación de pelets de mineral de hierro de grado \\ comercial. Los pelets fueron reducidos en hidrógeno hasta un $88 \%$ y carburizados en una mezcla de \\ gas $\mathrm{H}_{2}-\mathrm{CO}-\mathrm{CO}_{2}$, por $0,12,18,25$ y $32 \min$ a $700,800,900$ y $1000{ }^{\circ} \mathrm{C}$. Después del proceso de \\ reducción-carburización, los pelets se reoxidaron a $250,300,350$ y $400{ }^{\circ} \mathrm{C}$. Se encontró que el grado \\ de reoxidación disminuye con el incremento de la temperatura de reducción. Este efecto es más mar- \\ cado a las temperaturas de reoxidación de $250{ }^{\circ} \mathrm{C}$ y $300{ }^{\circ} \mathrm{C}$. La presencia de carburo de hierro $\left(\mathrm{Fe}_{3} \mathrm{C}\right)$ \\ fue más clara al incrementarse el tiempo de carburización cuando la reducción se llevó a cabo a 700 \\ y $800{ }^{\circ} \mathrm{C}$. Finalmente, se observó que el grado de reoxidación es mayor a medida que se incrementa \\ la temperatura de reoxidación.
}

Palabras clave: Reducción. Carburización. Reoxidación. Pelets de mineral de hierro.

\section{Reoxidation and carburization study of reduced iron ore pellets}

\begin{abstract}
The influence of carburization on the reoxidation of commercial iron ore pellets was studied. The pellets were $\mathrm{H}_{2}$-reduced up to $88 \%$ and then carburized in $\mathrm{H}_{2} / \mathrm{CO} / \mathrm{CO}_{2}$ gas mixture, for $0,12,18,25$ and $32 \mathrm{~min}$, at $700,800,900$ and $1000^{\circ} \mathrm{C}$. After the reduction-carburization process, the pellets were reoxidized at $250,300,350$ and $400{ }^{\circ} \mathrm{C}$. It was found out that the higher reduction temperature, the lower was the reoxidation degree. This effect was stronger at the reoxidation temperatures of 250 and $300{ }^{\circ} \mathrm{C}$. The presence of iron carbide $\left(\mathrm{Fe}_{3} \mathrm{C}\right)$ was more notorious when the reduction was carried out at 700 and $800{ }^{\circ} \mathrm{C}$, and the carburization time was increased. Finally, it was observed that the higher the reoxidation temperature, the higher was the reoxidation degree.
\end{abstract}

Keywords: Reduction. Carburization. Reoxidation. Iron ore pellets.

\section{INTRODUCCION}

La reoxidación del hierro esponja o hierro de reducción directa (DRI) se debe principalmente a su alta relación volumen/superficie, la cual está influida por la temperatura de reducción, por ejemplo, a mayor temperatura esta relación disminuye, según estudios realizados por I.G. Nixon (1). Es posible que las reacciones de reoxidación se lleven a cabo durante el almacenamiento y transporte, cuando la temperatura del hierro esponja en algunas áreas de la pileta se eleva por encima de la temperatura críti-

(•) Trabajo recibido el día 19 de febrero de 1998 y aceptado en su forma final el 17 de agosto de 1998.

(*) Dpt. of Mining and Metallurgical. McGill University. 3610 University St., H3A 2B2. Montreal (Quebec, Canadá).

(**) Centro de Investigación y Estudios Avanzados del IPN. Aptdo. postal 663. 25000, Saltillo (Coahuila, México).

(***) Instituto de Investigaciones Metalúrgicas. Universidad Michoacana de San Nicolás de Hidalgo. Aptdo. postal 52-B. Morelia (Mich., México). ca (por ej.: temperatura a la cual la reacción de combustión es espontánea con la atmósfera de oxígeno). La mayoría de las reacciones químicas de combustión involucradas en el fenómeno de reoxidación son exotérmicas, como se muestra en la tabla I. La energía libre de Gibbs y el calor de formación también se incluyen en la misma tabla (2).

Para evitar el fenómeno de la reoxidación se ha recurrido a técnicas tales como briqueteado, pasivación del hierro esponja por medio de la formación de una capa de óxido protector alrededor del pelet, o por recubrimientos con algunos compuestos orgánicos o inorgánicos para minimizar el contacto de la superficie del pelet con el oxígeno.

En la actualidad, existe poca información relacionada con el carácter pirofórico del hierro reducido. Iguchi et al. (3) establecieron que pelets de mineral de hierro, reducidos con hidrógeno a bajas temperaturas y ligeramente reoxidados a temperatura ambiente (usando un gas inerte con bajo potencial de oxígeno), son pasivados debido a la formación de una capa de óxido de hierro sobre la 
TABLA I.- Reacciones químicas envueltas en el proceso de reoxidación

TABLE I.-Chemical reactions involved in the reoxidation process

\begin{tabular}{|l|c|c|}
\hline \multicolumn{1}{|c|}{ Reacciones } & $\Delta H^{\mathrm{o}}{ }_{298}, \mathrm{~kJ} / \mathrm{mol}$ & $\Delta G^{\mathrm{o}}{ }_{298}, \mathrm{~kJ} / \mathrm{mol}$ \\
\hline $0,95<\mathrm{Fe}>+2\left(\mathrm{O}_{2}\right)=<\mathrm{Fe}_{0,95} \mathrm{O}>$ & $-264,002$ & $-244,632$ \\
$3<\mathrm{Fe}>+2\left(\mathrm{O}_{2}\right)=<\mathrm{Fe}_{3} \mathrm{O}_{4}>$ & $-1.103,120$ & $-1.011,515$ \\
$2<\mathrm{Fe}>+3 / 2\left(\mathrm{O}_{2}\right)=<\mathrm{Fe}_{2} \mathrm{O}_{3}>$ & $-815,023$ & $-740,400$ \\
$3<\mathrm{Fe}>+4\left(\mathrm{H}_{2} \mathrm{O}\right)=<\mathrm{Fe}_{3} \mathrm{O}_{4}>+4\left(\mathrm{H}_{2}\right)$ & $-149,460$ & $-88,520$ \\
$2<\mathrm{Fe}>+3\left(\mathrm{H}_{2} \mathrm{O}\right)=<\mathrm{Fe}_{2} \mathrm{O}_{3}>+3\left(\mathrm{H}_{2}\right)$ & $-72,847$ & $-50,548$ \\
\hline
\end{tabular}

superficie del pelet cuando se usa una mezcla $\mathrm{N}_{2}-$ $\mathrm{O}_{2}$ con menos del $1 \%$ de oxígeno. Por el contrario, cuando se excedió el $1 \%$ de oxígeno, la reoxidación se incrementó notoriamente. Sin embargo, se encontró que el tiempo necesario para la pasivación depende del potencial de oxígeno. Estos autores también mostraron que la velocidad de reoxidación fue controlada por difusión de oxígeno, a través de la capa límite y la película de óxido formada.

Por otro lado, Kamiya et al. (4) investigaron la influencia de la fuerza de compactación sobre la velocidad de reoxidación a diferentes temperaturas en briquetas de mineral de hierro obtenidas por la compactación de un polvo reducido con hidrógeno a $900{ }^{\circ} \mathrm{C}$. La reoxidación se realizó con una mezcla de $\mathrm{N}_{2}-\mathrm{O}_{2}$. Se encontró que la máxima velocidad de reoxidación fue alrededor de $400{ }^{\circ} \mathrm{C}$, atribuyendo este hecho a la formación de una capa delgada y densa reoxidada alrededor de las partículas a temperaturas mayores de $400{ }^{\circ} \mathrm{C}$. Estos autores también reportaron que la reoxidación de las briquetas obedece un comportamiento parabólico, y comienza a decrecer al incrementar la densidad de la capa reoxidada.

Sraku-Lartey et al. (5) estudiaron la reoxidación de pelets de hierro esponja en el rango de 60 a 600 ${ }^{\circ} \mathrm{C}$ reducidos con hidrógeno. Encontraron que la estructura del hierro esponja depende de la temperatura de reducción y que la reoxidación es controlada a su vez por dicha estructura y por la temperatura de reoxidación.

Por otro lado, A. Bandopadhyay et al. (6) estudiaron el mecanismo y los parámetros cinéticos de reoxidación en muestras de hierro esponja reoxidados a temperaturas altas y reducidos con monóxido de carbono, encontrando que la oxidación isotérmica en el rango de temperaturas de 447 a $597{ }^{\circ} \mathrm{C}$ obedece a una ley de primer orden, que se expresa de la siguiente manera:

$$
\mathrm{d} \alpha / \mathrm{d} t=K(1-\alpha)
$$

donde: $K=$ constante de velocidad

$\alpha=$ fracción reoxidada
Esta ley establece que la velocidad de reoxidación es proporcional a la cantidad de hierro sin reaccionar. También encontraron que el modelo cinético cambia a altas y bajas temperaturas de reoxidación.

La estructura del hierro reducido se caracteriza por su tamaño de poro, porosidad y área superficial específica. Esta estructura depende grandemente de la temperatura de reducción, y al mismo tiempo, la tendencia a la reoxidación depende de la estructura del pelet. Turkdogan et al. (7) establecen que esta tendencia decrece cuando la temperatura de reducción se incrementa, lo cual se atribuye a la mayor área superficial específica. Al parecer, la naturaleza de las materias primas también afecta la sensibilidad del hierro esponja a la reoxidación. Iguchi et al. (8) encontraron que la magnetita se reoxida más que la hematita después de la reducción, y que la limonita tiene alta tendencia a la reoxidación.

El-Geassy et al. (9) estudiaron la cinética y el mecanismo de reducción y reoxidación de compactos de hematita reducidos con hidrógeno de $700 \mathrm{a}$ $1.000{ }^{\circ} \mathrm{C}$ y reoxidados en aire de 200 a $800{ }^{\circ} \mathrm{C}$. Encontraron que el proceso de reducción en la etapa inicial es controlado por difusión gaseosa, mientras que la velocidad del proceso en las etapas posteriores puede ser controlado por un mecanismo combinado de difusión gaseosa en la capa producto y una reacción química interfacial. La reoxidación en las etapas iniciales se controló por reacción química en la interfase, mientras que en las etapas final e intermedia, la velocidad del proceso se controló por difusión en estado sólido.

El presente trabajo estudia la reoxidación de pelets de mineral de hierro reducidos y carburizados previamente en mezclas de gas $\mathrm{H}_{2} / \mathrm{CO} / \mathrm{CO}_{2}$ a diferentes temperaturas.

\section{PARTE EXPERIMENTAL}

\subsection{Materiales}

En el presente trabajo, se utilizaron pelets de mineral de hierro de grado comercial de las minas de Peña Colorada, Colima, México. En las tablas II y 
TABLA II.- Composición química de los pelets sinterizados

TABLE II.-Chemical composition of sintered pellets

\begin{tabular}{|c|c|c|c|c|c|c|}
\hline $\mathrm{Fe}_{\text {total }}$ & $\mathrm{Fe}^{2+}$ & $\mathrm{SiO}_{2}$ & $\mathrm{CaO}$ & $\mathrm{MgO}$ & $\mathrm{Al}_{2} \mathrm{O}_{3}$ & $\mathrm{~S}$ \\
\hline 65,80 & 0,35 & 2,36 & 1,95 & 0,51 & 1,06 & 0,012 \\
\hline
\end{tabular}

III se muestran la composición química y algunas propiedades físicas de los pelets empleados, respectivamente.

\subsection{Pruebas de reducción}

La reducción se controló a través de los cambios de peso en función del tiempo. La reducibilidad de las muestras se realizó en un analizador termogravimétrico Cahn (TGA-171), utilizando un proceso isotérmico a $700,800,900$ y $1.000{ }^{\circ} \mathrm{C}$, bajo una mezcla de gas $\mathrm{H}_{2}-\mathrm{CO}-\mathrm{CO}_{2}$ (Tabla IV). La velocidad del flujo volumétrico de los gases fue de $180 \mathrm{~mL} / \mathrm{min}$.

El grado de reducción se calculó a partir de la pérdida de peso con la siguiente fórmula:

$$
\text { Reducibilidad }=\Delta W / W_{1} \times 100 \%
$$

donde: $\Delta W=$ peso de la muestra antes de la reducción-peso de la muestra después de la reducción $(\mathrm{g})$.

$W_{1}=$ pérdida de peso correspondiente al $100 \%$ de reducción $(\mathrm{g})$.

Para cada experimento de reducción, se colocaron 3 pelets en una canastilla de níquel metálico y se introdujeron en el horno de la termobalanza. Posteriormente, se calentaron hasta la temperatura de reducción bajo un flujo de nitrógeno; una vez que la temperatura de reducción alcanzó el flujo de gas inerte se cambió por uno de hidrógeno hasta que se alcanzó un grado de reducción del $88 \%$, entonces se hizo pasar una mezcla gaseosa de $\mathrm{H}_{2}$ $\mathrm{CO}-\mathrm{CO}_{2}$ a diferentes tiempos $(12,18,25$ y $32 \mathrm{~min})$, para promover la carburización. Cuando el proceso

TABLA III.- Propiedades físicas de los pelets sinterizados

TABLE III.- Physical properties of sintered pellets
Porosidad

Índice al tambor

Índice a la abrasión

Densidad aparente

Diámetro medio de pelet
$24,4 \%$

$93,10 \%$

$5,15 \%$

$2,25 \mathrm{~g} / \mathrm{cm}^{3}$

$0,90 \mathrm{~cm}$ de reducción finalizó, las muestras se enfriaron en una atmósfera de nitrógeno. Las condiciones usadas en el proceso de reducción se muestran en la tabla IV. La microestructura de los pelets reducidos y reoxidados se analizó por difracción de rayos-X, microscopía óptica y microscopía electrónica de barrido.

\subsection{Pruebas de reoxidación}

La reoxidación se controló a través de los cambios de peso en función del tiempo. La reoxidación de las muestras se llevó a cabo en el sistema TGA antes mencionado, con un proceso isotérmico, a $250,300,350$ y $400{ }^{\circ} \mathrm{C}$, bajo un flujo de aire. La velocidad del flujo volumétrico fue de $200 \mathrm{~mL} / \mathrm{min}$. El grado de reoxidación se calculó de la ganancia de peso usando la fórmula:

$$
\text { Reoxidación }=\Delta W / W_{2} \times 100 \%
$$

donde: $\Delta W=$ peso de la muestra después de la reoxidación-peso de la muestra antes de la reoxidación $(\mathrm{g})$.

$W_{2}$ = ganancia de peso correspondiente al $100 \%$ de reoxidación $(\mathrm{g})$.

Por cada experimento de reoxidación, se colocaron dos pelets en una canastilla de níquel y se introdujeron en el horno de la termobalanza. Posteriormente, se calentaron hasta la temperatura de reoxidación bajo un flujo de gas nitrógeno, el cual se cambió por un flujo de aire una vez que se alcanzó la temperatura de reoxidación. Las muestras se mantuvieron bajo estas condiciones por $120 \mathrm{~min}$ y después se enfriaron hasta temperatura ambiente en nitrógeno. La microestructura de los pelets reoxidados se analizó por microscopía óptica y rayos-X.

TABLA IV- Valores de los parámetros usados en el proceso de reducción-carburización

TABLE IV.-Parameters values used in the reductioncarburization process

\begin{tabular}{|c|c|c|}
\hline $\begin{array}{c}\text { Temperatura } \\
\text { reducción- } \\
\text { carburización, }{ }^{\circ} \mathrm{C}\end{array}$ & $\begin{array}{c}\text { Mezcla de gas } \\
\% \mathrm{H}_{2} / \% \mathrm{CO} / \\
\% \mathrm{CO}_{2}\end{array}$ & $\begin{array}{c}\text { Tiempo } \\
\text { carburización, } \\
\text { min }\end{array}$ \\
\hline 700 & $14 / 77 / 9$ & $0,12,18,25,32$ \\
800 & $14 / 77 / 9$ & $0,12,18,25,32$ \\
900 & $13 / 86 / 1$ & $0,12,18,25,32$ \\
1.000 & $10 / 89 / 1$ & $0,12,18,25,32$ \\
\hline
\end{tabular}

Rev. Metal. Madrid, 34 (6), 1998 461 


\section{RESULTADOS Y DISCUSIÓN}

Los pelets sinterizados se redujeron con hidrógeno a $700,800,900$ y $1.000{ }^{\circ} \mathrm{C}$, hasta alcanzar un $88 \%$ de reducción y, posteriormente, carburizados con mezclas de $\mathrm{CO}-\mathrm{H}_{2}-\mathrm{CO}_{2}$ por $12,18,25$ y $32 \mathrm{~min}$, con el objeto de obtener pelets con diferentes cantidades de la fase carburo de hierro y conteniendo a la vez un porcentaje de reducción comparable al que se obtiene industrialmente.

\subsection{Efecto de la temperatura en la reducción- carburización}

Las figuras 1 y 2 muestran las curvas de velocidad de pérdida de peso obtenidas en los experimentos de reducción-carburización para las temperaturas de reducción de 700 y $1.000{ }^{\circ} \mathrm{C}$ y distintos tiempos de carburización. Como se puede observar, en la etapa inicial de reducción (primeros $20 \mathrm{~min}$ ) existe una velocidad de pérdida de peso mayor que en la etapa posterior de la reducción, observándose esto en todas las temperaturas aquí estudiadas. Asimismo, se aprecia que la velocidad de reducción se incrementa a medida que la temperatura aumenta. Esto se debe, a que la mayor parte de las reacciones que intervienen en la reducción son endotérmicas y se ven favorecidas con la temperatura. En la etapa de carburización, se observa que, conforme se incrementa el tiempo, la velocidad disminuye hasta llegar en algunas temperaturas a registrarse una ganancia en el peso que se atribuye a la depositación de carbono en el pelet y a la posterior carburización, lo cual concuerda con algunos trabajos (10 y 11).

La ganancia de peso está favorecida tanto por el tiempo de carburización como por el contenido de

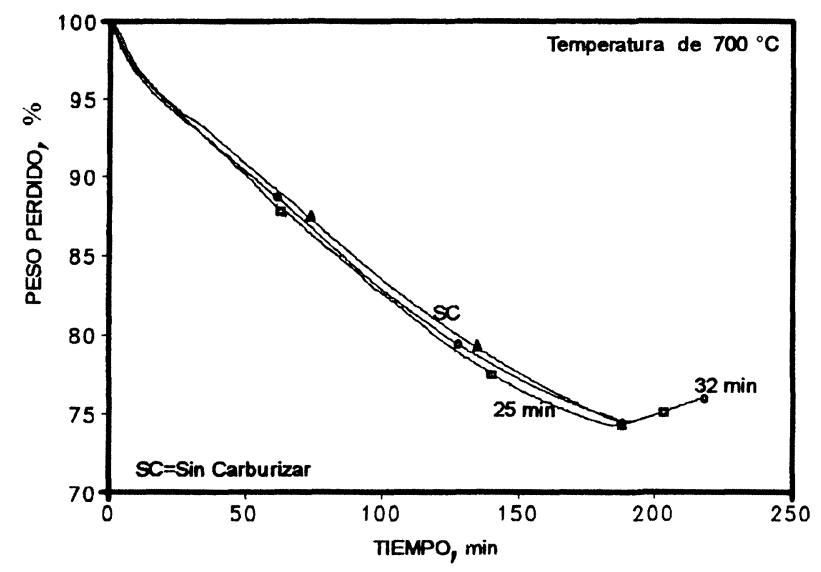

FIG. 1.- Curvas de velocidad de reducción a $700{ }^{\circ} \mathrm{C}$ y diferentes tiempos de carburización.

FIG. 1.- Reduction rate curves at $700{ }^{\circ} \mathrm{C}$ and different carburization times.

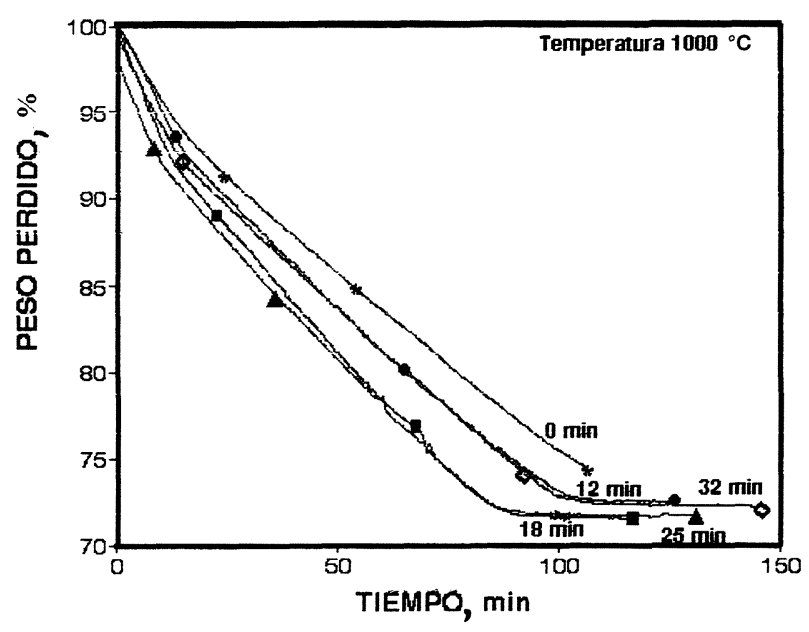

FIG. 2- Curvas de velocidad de reducción a $1.000^{\circ} \mathrm{C}$ y diferentes tiempos de carburización.

\section{FIG. 2.- Reduction rate curves at $1.000{ }^{\circ} \mathrm{C}$ and different carburization times.}

monóxido de carbono de la mezcla de gases, así como por la disminución de la temperatura. Estos resultados están en concordancia con lo encontrado por algunos investigadores $(12,13,14$ y 15$)$, que señalan que la disociación del monóxido de carbono por la reacción de Boudouard ocasiona la formación de depósitos de carbono, la cual se incrementa al disminuir la temperatura y con una concentración elevada de monóxido de carbono. La figura 3 muestra la velocidad de reducción-carburización a diferentes temperaturas de reducción para un tiempo de carburización de $32 \mathrm{~min}$. Se puede observar que la velocidad de reducción aumenta conforme se incrementa la temperatura, de tal forma que se alcanza el

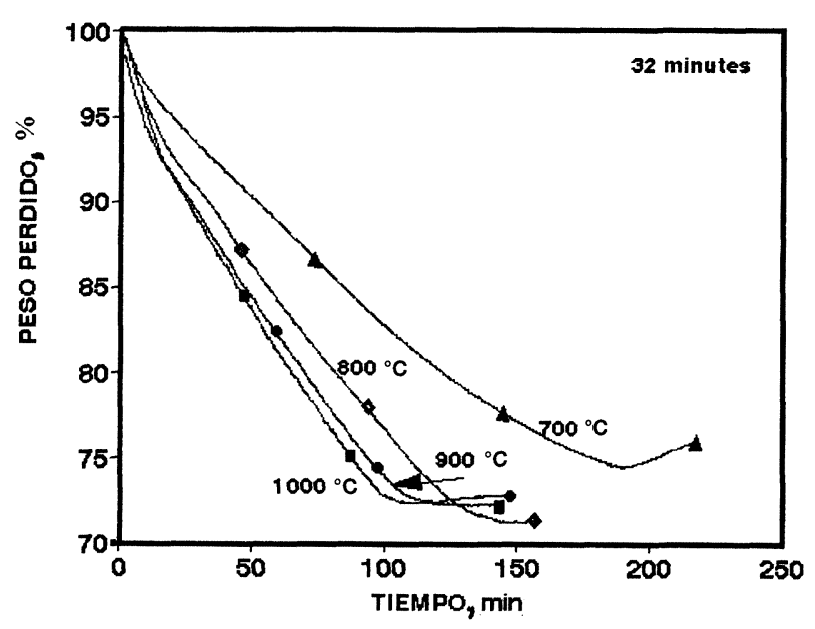

FIG. 3.- Curvas de velocidad de reducción a diferentes temperaturas y $32 \mathrm{~min}$ de carburización.

FIG. 3.- Reduction rate curves at different reduction temperatures and 32 min carburization time. 
grado de reducción establecido en un tiempo menor. Por otro lado, en la etapa de carburización se puede observar cómo a medida que la temperatura disminuye, la ganancia en peso se hace mayor, sobre todo a 700 y $800{ }^{\circ} \mathrm{C}$.

Espectros de rayos-X mostraron un incremento en la intensidad de los picos correspondientes al carburo de hierro al incrementar el tiempo de carburización, y una disminución de estos al incrementarse la temperatura de reducción. La tabla V muestra que el contenido de carbón total aumenta con el tiempo de carburización y disminuye al incrementarse la temperatura de reducción.

\subsection{Efecto de la temperatura en la velocidad de reoxidación}

Los pelets reducidos con hidrógeno y carburizados con mezclas de $\mathrm{CO}-\mathrm{H}_{2}-\mathrm{CO}_{2}$ a distintos tiempos en el rango de temperatura de 700 a $1000{ }^{\circ} \mathrm{C}$, fueron reoxidados en aire a las temperaturas de 250, 300, 350 y $400{ }^{\circ} \mathrm{C}$. La figura 4 muestra las curvas de velocidad de reoxidación obtenidas para pelets reducidos a $800{ }^{\circ} \mathrm{C}$ sin carburizar y carburizados durante 32 min para las distintas temperaturas de reoxidación estudiadas. Las curvas de reoxidación en general, consisten en tres etapas: una primera, en el estado inicial, caracterizándose por una apreciable pendiente en la curva, una segunda, mostrando un marcado decaimiento en esta pendiente y un tercer estado con valor de la pendiente cercano a cero. La longitud y el tiempo de cada una de estas etapas varía de acuerdo a las condiciones de cada experimento como puede observarse en la figura 4. Generalmente, todas las curvas presentan una mayor velocidad de reoxidación en la etapa inicial del proceso, observándose que en la etapa final la velocidad de reoxidación es muy lenta. Este comportamiento se debe a que en la primera etapa del proceso de reoxidación se forma una capa densa de óxido alrededor del pelet y de los granos, lo cual

TABLA V.- Porcentaje de carbón total en los pelets obtenidos de la reducción-carburización

TABLE V.-- Total carbon percent in the pellets after reduction-carburization process

\begin{tabular}{|c|c|c|c|c|}
\hline \multirow{2}{*}{$\begin{array}{l}\text { Temperatura de } \\
\text { reducción, }{ }^{\circ} \mathrm{C}\end{array}$} & \multicolumn{4}{|c|}{$\begin{array}{l}\text { Tiempo de carburización, } \\
\text { min }\end{array}$} \\
\hline & 12 & 18 & 25 & 32 \\
\hline 70 & 0,653 & 0,872 & 1,237 & 1,910 \\
\hline 80 & 0,2 & 0,4 & 1,144 & 1,371 \\
\hline 900 & 0,110 & 0,204 & 0,413 & 0,690 \\
\hline 1.000 & 0,080 & 0,102 & 0,294 & 0,324 \\
\hline
\end{tabular}
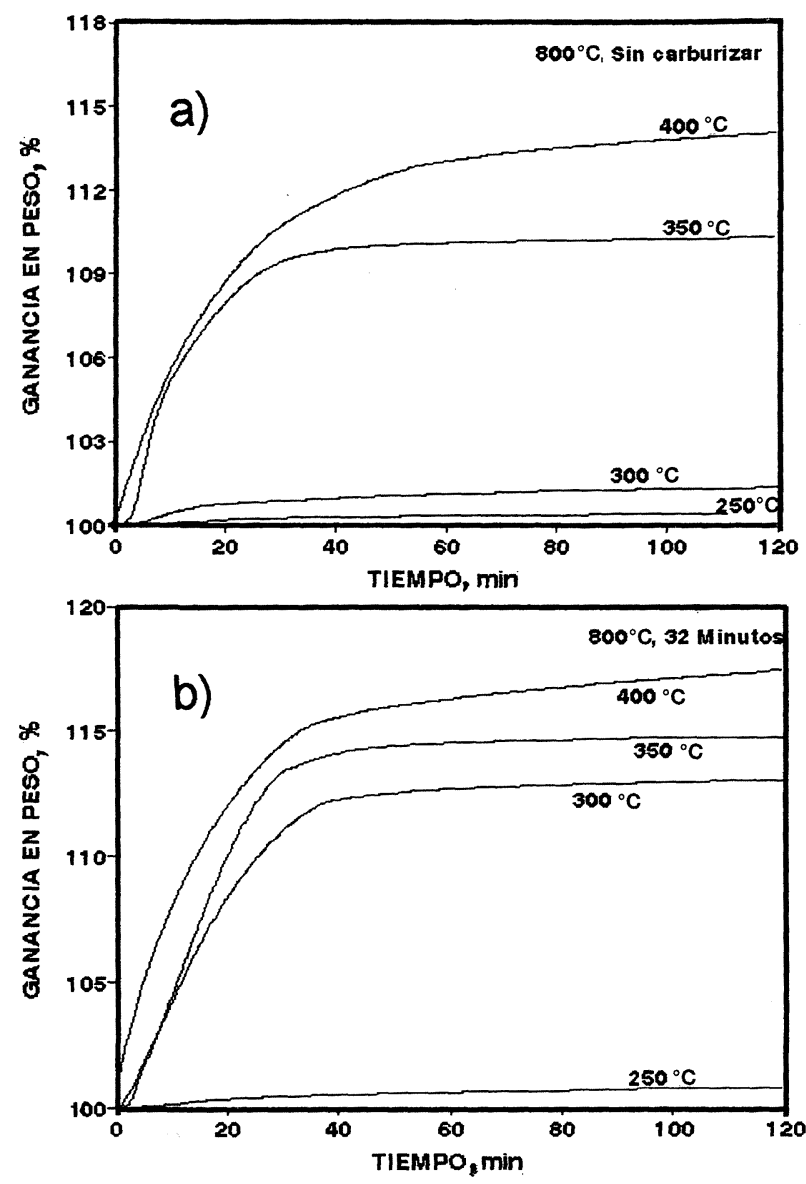

FIG. 4.- Curvas de velocidad de reoxidación para los pelets reducidos a $800{ }^{\circ} \mathrm{C}$ a diferentes temperaturas de reoxidación: a) sin carburizar y b) $32 \mathrm{~min}$ de carburización.

FIG. 4.- Reoxidation rate curves for $800{ }^{\circ} \mathrm{C}$ reduced pellets at different reoxidation temperatures. a) non-carburized pellets and b) 32 min carburization time.

contribuye a la pasivación del proceso de reoxidación del pelet. En su mayoría, la velocidad de reoxidación aumenta con la temperatura de reoxidación.

\subsection{Efecto de la temperatura de reoxidación}

La figura 5 muestra la influencia de la temperatura de reoxidación en el grado de reoxidación para el material reducido a las diferentes temperaturas de reducción y los tiempos de carburización aquí estudiados. Como se puede observar, el grado de reoxidación es mayor a medida que la temperatura de reoxidación aumenta independientemente de la temperatura de reducción y tiempo de carburización estudiados; sin embargo, a la temperatura de 250 ${ }^{\circ} \mathrm{C}$, se observa una reoxidación incipiente en las muestras reducidas a 700 y $800^{\circ} \mathrm{C}$, incrementándose a partir de esta última temperatura. Para los 

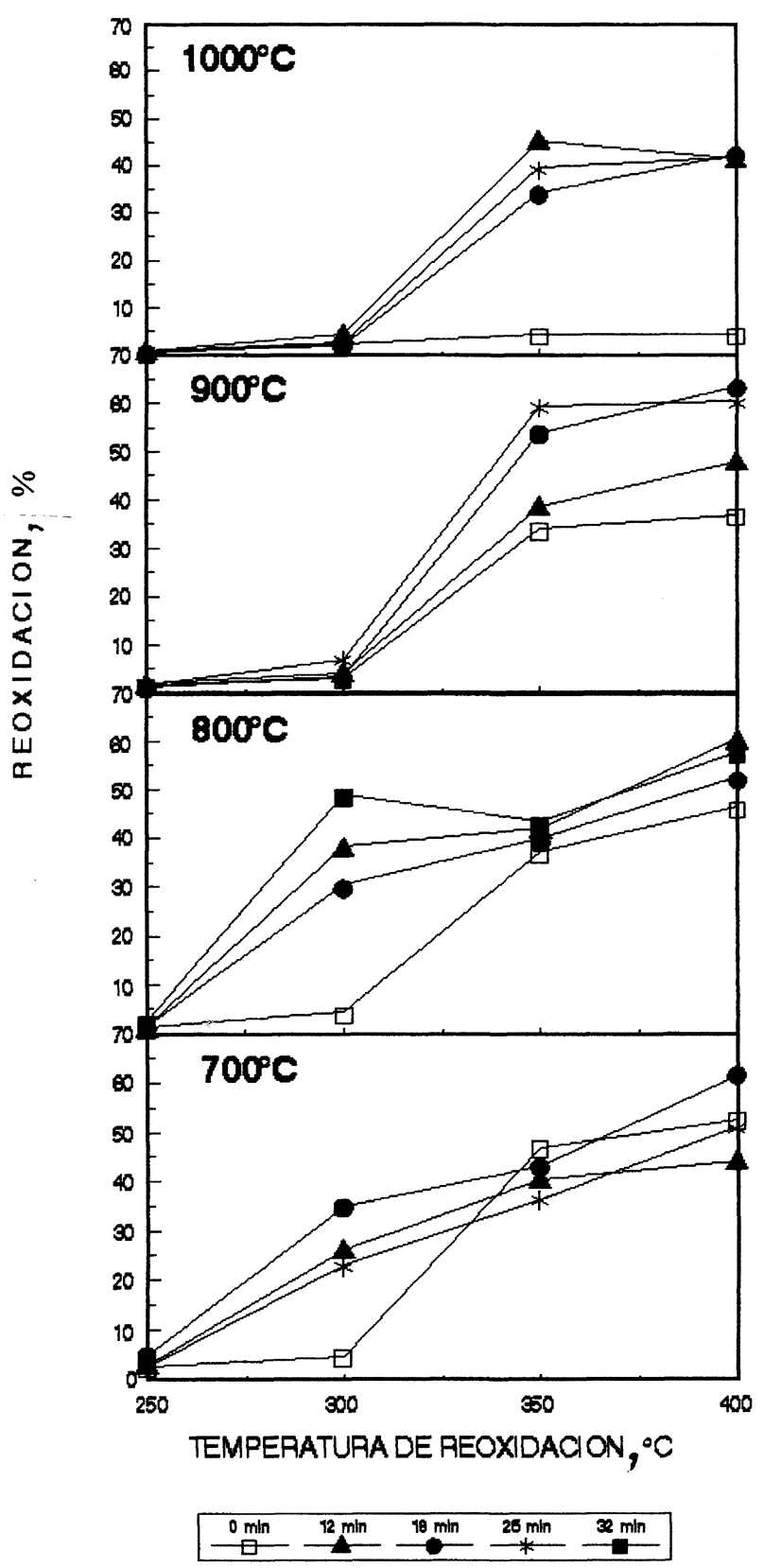

FIG. 5.- Influencia de la temperatura en el grado de reoxidación a las diferentes temperaturas de reducción y tiempos de carburización

FIG. 5.- Influence of reoxidation temperature on the reoxidation degree at different reduction temperatures and carburization times.

pelets reducidos a 900 y $1.000{ }^{\circ} \mathrm{C}$, el grado de reoxidación es bajo hasta una temperatura de oxidación de $300{ }^{\circ} \mathrm{C}$, presentándose un marcado incremento a temperaturas superiores.

Por otro lado, se puede observar también que a las temperaturas de reducción de 700 y $800{ }^{\circ} \mathrm{C}$, la influencia del tiempo de carburización en el grado de reoxidación obtenido no es muy clara; sin embargo, a 900 y $1.000{ }^{\circ} \mathrm{C}$ se aprecia claramente que el tiempo de carburización provoca un aumento en el grado de reoxidación, sobre todo a las temperaturas de reoxidación de 350 y $400{ }^{\circ} \mathrm{C}$. Este comportamiento se atribuye a que a bajas temperaturas (700 y $800{ }^{\circ} \mathrm{C}$ ), la cantidad de poros formada por el monóxido de carbono durante el proceso de reducción-carburización es muy pequeña. En cambio, a temperaturas de reducción altas $\left(900\right.$ y $\left.1.000{ }^{\circ} \mathrm{C}\right)$, la porosidad de las muestras reducidas solamente con hidrógeno se ve afectada por el tiempo de carburización con monóxido de carbono, lo cual se constató realizando un análisis cuantitativo de la porosidad de los pelets reducidos y carburizados a las diferentes temperaturas y tiempos de carburización, mediante la técnica de conteo por puntos, encontrando que a 900 y $1.000^{\circ} \mathrm{C}$ la porosidad es directamente proporcional al tiempo de carburización; contrario a lo que sucede a 700 y $800{ }^{\circ} \mathrm{C}$, ya que en general, los pelets son muy porosos. La tabla VI muestra los resultados de la porosidad obtenidos en los pelets reducidos a 700 y $900{ }^{\circ} \mathrm{C}$ a los diferentes tiempos de carburización. De aquí, se concluye que la porosidad es el factor más importante en el control de la reoxidación y en el caso particular de pelets reducidos a temperaturas altas $(900$ y 1.000 ${ }^{\circ} \mathrm{C}$ ), la porosidad se incrementa conforme mayor es el tiempo del proceso de reducción-carburización.

Con el propósito de lograr una visión generalizada del proceso de reoxidación y la influencia de la temperatura y el tiempo de carburización, la figura 6 muestra una gráfica del promedio de los resultados obtenidos para el grado de reoxidación alcanzado a las diferentes temperaturas de reoxidación y tiempos de carburización, promediando los resultados obtenidos para el porcentaje de reoxidación a las diferentes temperaturas de reducción. Como se puede observar, el grado de reoxidación aumenta con la temperatura. Cabe destacar también, que en las muestras de pelets reoxidados sin carburizar, el grado de reoxidación alcanzado es menor que el obtenido para los pelets carburizados a los distintos tiempos aquí estudiados y, al analizar las muestras, se nota una menor porosidad en estas, por lo que se presume que el factor que más influye en este comportamiento es la porosidad.

TABLA VI.- Porcentaje de porosidad en los pelets obtenidos de la reducción-carburización

TABLE VI.- Percent porosity in the pellets after reduction-carburization process

\begin{tabular}{|c|c|c|c|c|c|}
\hline \multirow{2}{*}{$\begin{array}{c}\text { Temperatura de } \\
\text { reducción, }\end{array}{ }^{\circ} \mathrm{C}$} & \multicolumn{5}{|c|}{ Tiempo de carburización, } \\
& 0 & 12 & 18 & 25 & 32 \\
\cline { 2 - 6 } & 32,18 & 35,73 & 33,41 & 33,59 & 36,62 \\
700 & 14,96 & 16,73 & 23,97 & 29,83 & 32,15 \\
\hline
\end{tabular}




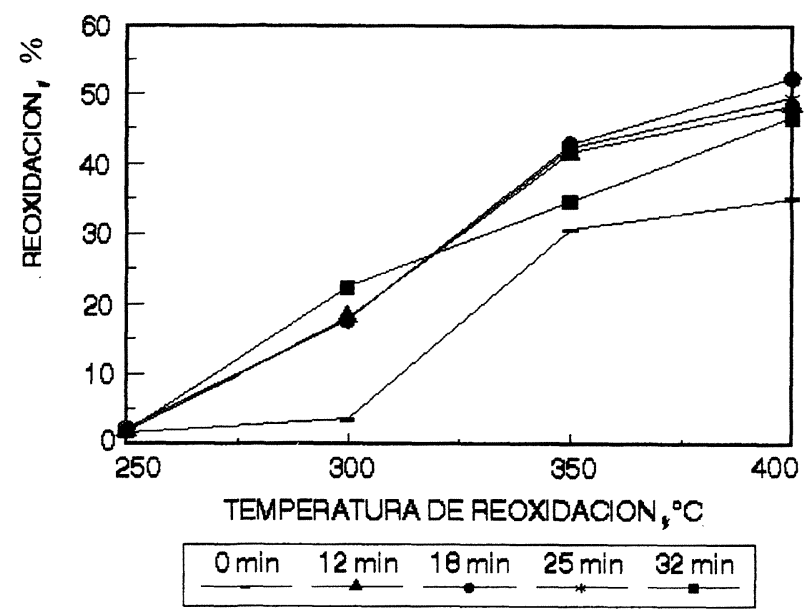

FIG. 6.- Grado de reoxidación frente a temperatura de reoxidación a los diferentes tiempos de carburización, promediando las diferentes temperaturas de reducción.

FIG. 6.- Reoxidation percent vs reoxidation temperature for different carburization times, averaged at different reduction temperatures.

\subsection{Influencia de la temperatura de reducción en la reoxidación}

Los pelets reducidos y carburizados a las temperaturas y tiempos ya mencionados fueron reoxidados a las temperaturas previamente programadas para observar la influencia que tiene la temperatura de reducción sobre el grado de reoxidación.

La figura 7 muestra la influencia de la temperatura de reducción en el grado de reoxidación, donde se puede observar una disminución de este último, para las diferentes temperaturas y tiempos de carburización estudiados, a medida que la temperatura de reducción aumenta.

El comportamiento encontrado en el porcentaje de reoxidación al incrementar la temperatura de reducción, se atribuye principalmente a la morfología del hierro esponja presente en los pelets al ser reducidos a las diferentes temperaturas de reducción-carburización. La estructura del mineral de hierro reducido se caracteriza por el tamaño de poro, porosidad y área superficial específica, donde la temperatura de reducción tiene un gran efecto sobre esta estructura, incrementando la tendencia a la reoxidación cuando la temperatura de reducción disminuye; esto es debido al aumento en el área superficial específica como mostraron Tukdogan et al. (11).

Por otro lado, al aumentar la temperatura de reducción disminuye la relación existente entre volumen/superficie y con esto la tendencia a la reoxidación del material, de acuerdo a lo encontrado por I.G. Nixon (1). Además, existe una mayor compactación en la estructura del pelet a medida que se

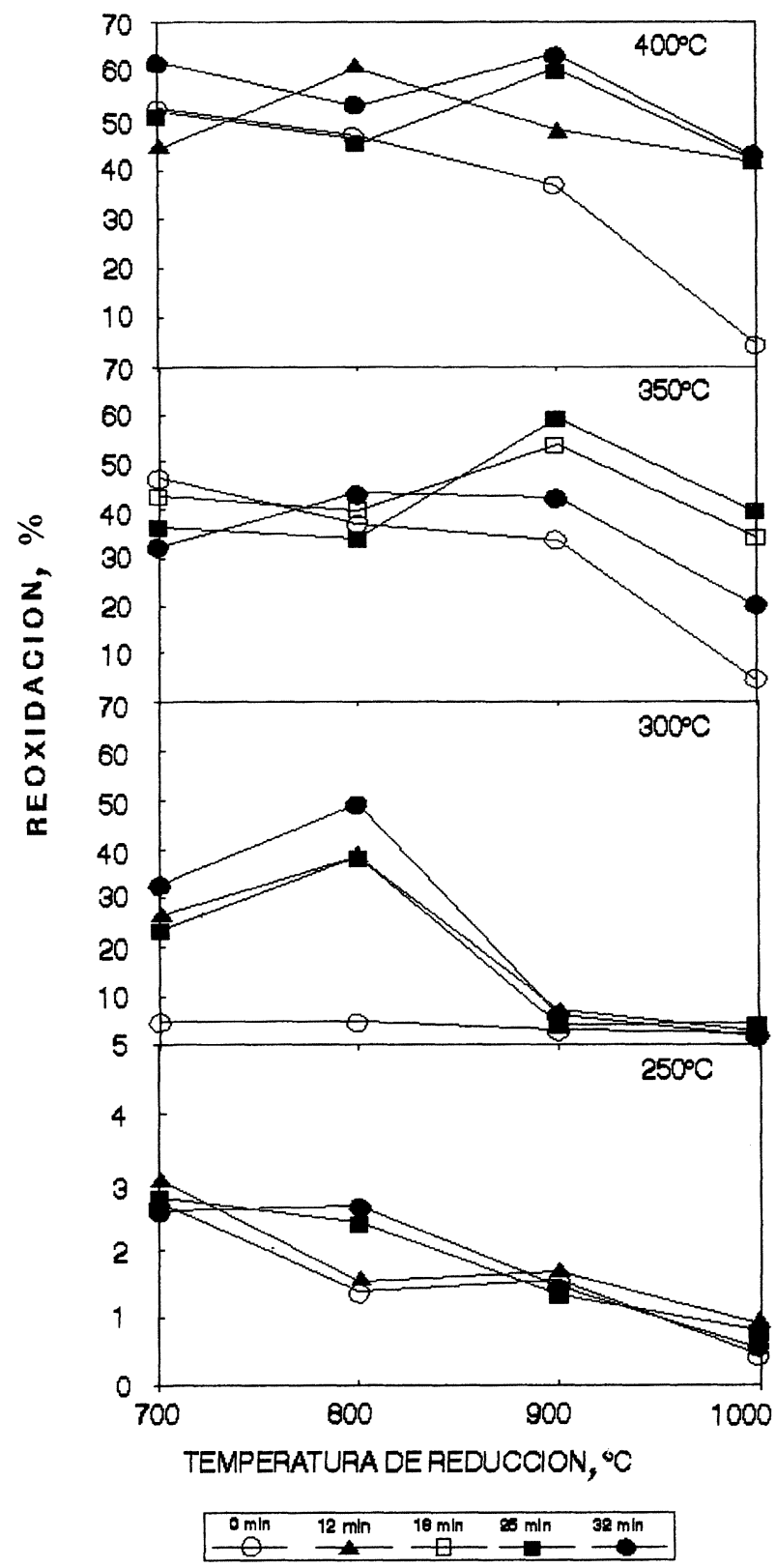

FIG. 7.- Influencia de la temperatura de reducción en el grado de reoxidación a las diferentes temperaturas de reoxidación y tiempos de carburización.

FIG. 7.- Influence of reduction temperature on the reoxidation degree at different reoxidation temperatures and carburization times.

incrementa la temperatura de reducción, como se puede observar en la figura 8 , donde se muestran las microestructuras obtenidas mediante microscopía electrónica de barrido de pelets reducidos a las diferentes temperaturas._Se observa una morfología muy similar a las temperaturas de reducción de 700 y $800{ }^{\circ} \mathrm{C}$, y lo mismo para 900 y $1.000{ }^{\circ} \mathrm{C}$, pero estas morfologías son diferentes en los dos intervalos de temperatura considerados. Se aprecia también que entre 700 y $800{ }^{\circ} \mathrm{C}$ existen grandes cavidades y granos pequeños, y en el intervalo 900-1.000 

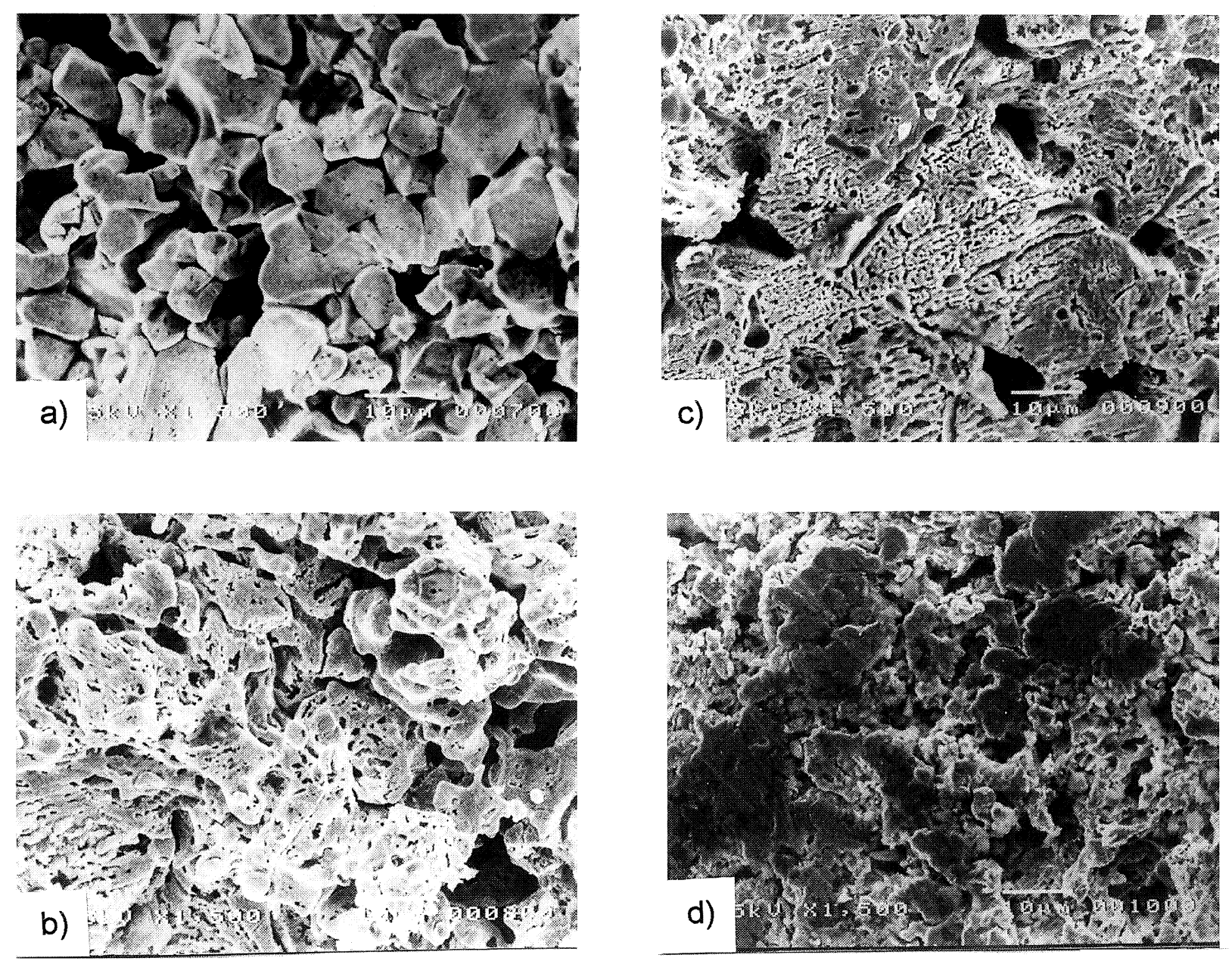

FIG. 8.- Micrografías obtenidas por microscopía electrónica de barrido de los pelets reducidos sin carburizar a a) $700{ }^{\circ} \mathrm{C}$. b) $800{ }^{\circ} \mathrm{C}$, c) $900{ }^{\circ} \mathrm{C}$ y d) $1.000{ }^{\circ} \mathrm{C}$.

FIG. 8. - SEM of non-carburized pellets at a) $\left.700{ }^{\circ} \mathrm{C}, b\right) 800^{\circ} \mathrm{C}$, c) $900{ }^{\circ} \mathrm{C}$ and d) $1.000{ }^{\circ} \mathrm{C}$.

${ }^{\circ} \mathrm{C}$ se inicia un proceo de sinterización. Al aumentar la temperatura de reducción de los pelets, se puede observar un mayor crecimiento de grano en la estructura, así como una mayor compactación y una disminución en forma muy notoria de la porosidad.

En general, se puede afirmar que la porosidad disminuye cuando la temperatura de reducción incrementa y con ello el grado de reoxidación que se alcanza es menor, como se puede observar en la figura 9 , donde se presenta una gráfica del promedio de los valores obtenidos en la reoxidación a las diferentes temperaturas de reducción y tiempos de carburización, promediando los resultados obtenidos a las diferentes temperaturas de reoxidación estudiadas. Esta tendencia, como ya se mencionó, es más clara en el caso de las muestras sin carburizar debido a que presentan un menor contenido de porosidad que las muestras carburizadas, en las cuales la presencia de monóxido de carbono durante la carburización contribuye a que exista un incremento de la porosidad.

\subsection{Análisis de los pelets reoxidados}

Los pelets reoxidados a las distintas temperaturas fueron analizados mediante un estudio metalográfico y de rayos-X, observándose un proceso de reoxidación del tipo homogéneo en todo el pelet; se aprecia, además, en los pelets reoxidados a 350 y $400{ }^{\circ} \mathrm{C}$ una porosidad abierta, lo que ocasiona un mayor grado de reoxidación. En las figuras 10a y $10 \mathrm{~b}$, se presentan micrografías, tomadas por microscopía óptica, de los pelets reoxidados a 250 y $400{ }^{\circ} \mathrm{C}$, en las cuales se puede observar el tipo de reoxidación homogénea en el pelet.

Los estudios de rayos- $X$ de los pelets reoxidados revelan las fases presentes en la microestructura del pelet, las cuales consisten básicamente en magnetita y hierro en los pelets reoxidados a las temperaturas de 250 y $300{ }^{\circ} \mathrm{C}$, y de magnetita, hematita y hierro en los pelets reoxidados a las temperaturas de 350 and 400 ${ }^{\circ} \mathrm{C}$, notándose la ausencia de wustita en todos los pelets reoxidados, como se muestra en la figura 11 . 


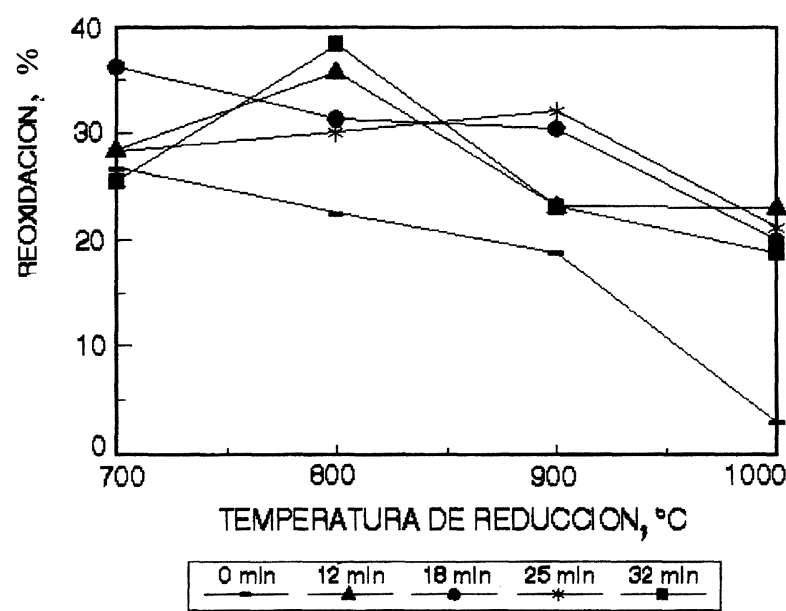

Fig. 9.- Grado de reoxidación frente a temperatura de reducción para los diferentes tiempos de carburizacion, promediando las diferentes temperaturas de reoxidación.

FIG. 9.- Reoxidation percent vs reduction temperature for different carburization times, averaged at different reoxidation temperatures.

\subsection{Ajuste matemático de los resultados}

Los datos experimentales se ajustaron mediante un análisis de regresión múltiple, con el fin de establecer alguna relación matemática y al mismo tiempo sugerir una tendencia más clara del grado de reoxidación con respecto a las variables que intervienen en el proceso de reoxidación, tales como temperatura de reducción, temperatura de reoxidación y tiempo de carburización. Se obtuvo la siguiente ecuación:

$\%$ reox. $=-0,0521 T_{1}+0,000245 T_{2}^{2}-0,1462 \mathrm{t}$

donde: $\%$ reox. $=$ Porcentaje de reoxidación.

$T_{1} \quad=$ Temperatura de reducción $\left({ }^{\circ} \mathrm{C}\right)$.

$T_{2} \quad=$ Temperatura de reoxidación $\left({ }^{\circ} \mathrm{C}\right)$.

$t=$ Tiempo de carburización (min).

Con un coeficiente de correlación de 0,905.

La ecuación anterior establece que el porcentaje de reoxidación disminuye en forma lineal con la temperatura de reducción y se incrementa de forma cuadrática con la temperatura de reoxidación, lo cual concuerda con lo encontrado en las figuras 6 y 9. Al mismo tiempo, se observa que el porcentaje de reoxidación para las condiciones utilizadas en este caso depende primeramente de la temperatura de reducción, posteriormente de la temperatura de reoxidación, y en última instancia del tiempo de
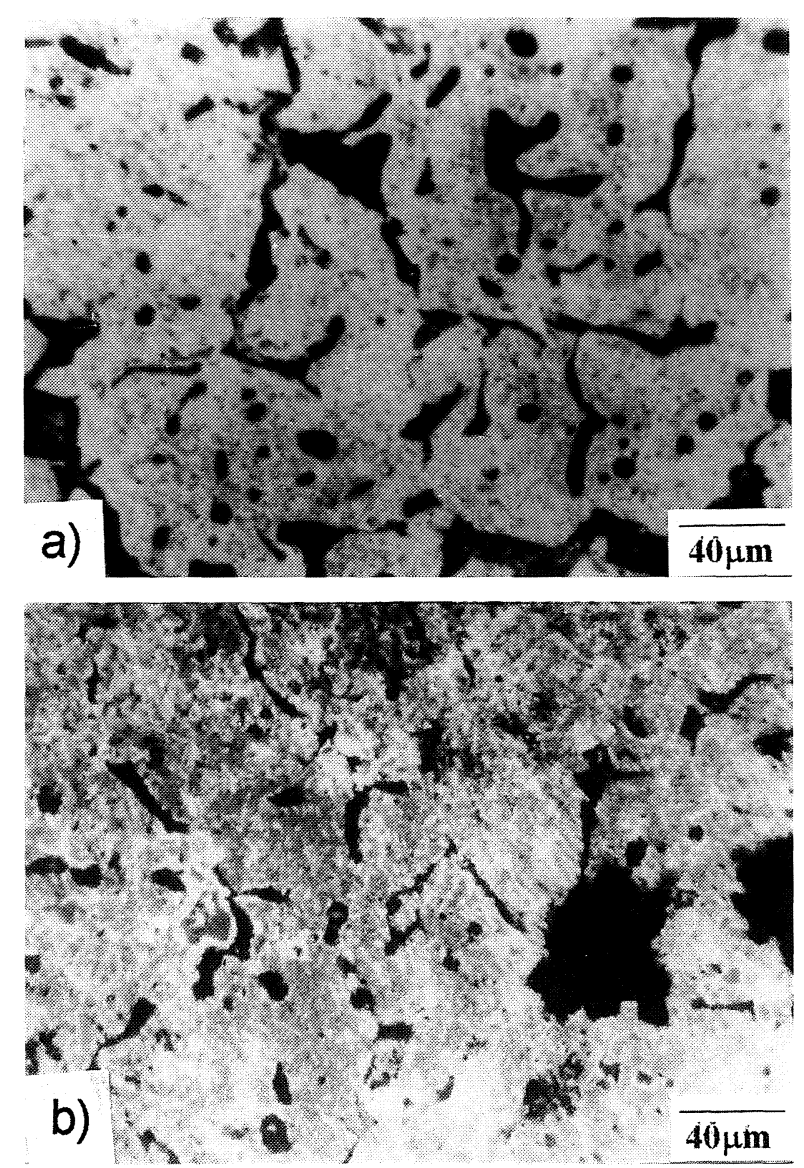

FIG. 10.- Micrografías de pelets sin carburizar reducidos a $800{ }^{\circ} \mathrm{C}$ y reoxidados a a) $250{ }^{\circ} \mathrm{C}$ y b) $400{ }^{\circ} \mathrm{C}$.

FIG. 10.- Micrographs of non-carburized pellets, reduced at $800^{\circ}$ and reoxidized at a) $250{ }^{\circ} \mathrm{C}$ and b) $400{ }^{\circ} \mathrm{C}$.

carburización. Los valores menores de reoxidación encontrados en el presente trabajo, se registraron a 250 y a $300{ }^{\circ} \mathrm{C}$ en el caso de las temperaturas de reducción de 900 y $1.000{ }^{\circ} \mathrm{C}$, para todos los tiempos de carburización estudiados.

\section{CONCLUSIONES}

Del análisis de los resultados contenidos en el presente trabajo, se desprenden las siguientes conclusiones:

- La carburización de los pelets se incrementa con el tiempo y disminuye conforme la temperatura de reducción aumenta.

- La reoxidación es casi nula para todos los pelets reducidos a las diferentes temperaturas y tiempos de carburización cuando el proceso de reoxidación se lleva a cabo a $250{ }^{\circ} \mathrm{C}$. 

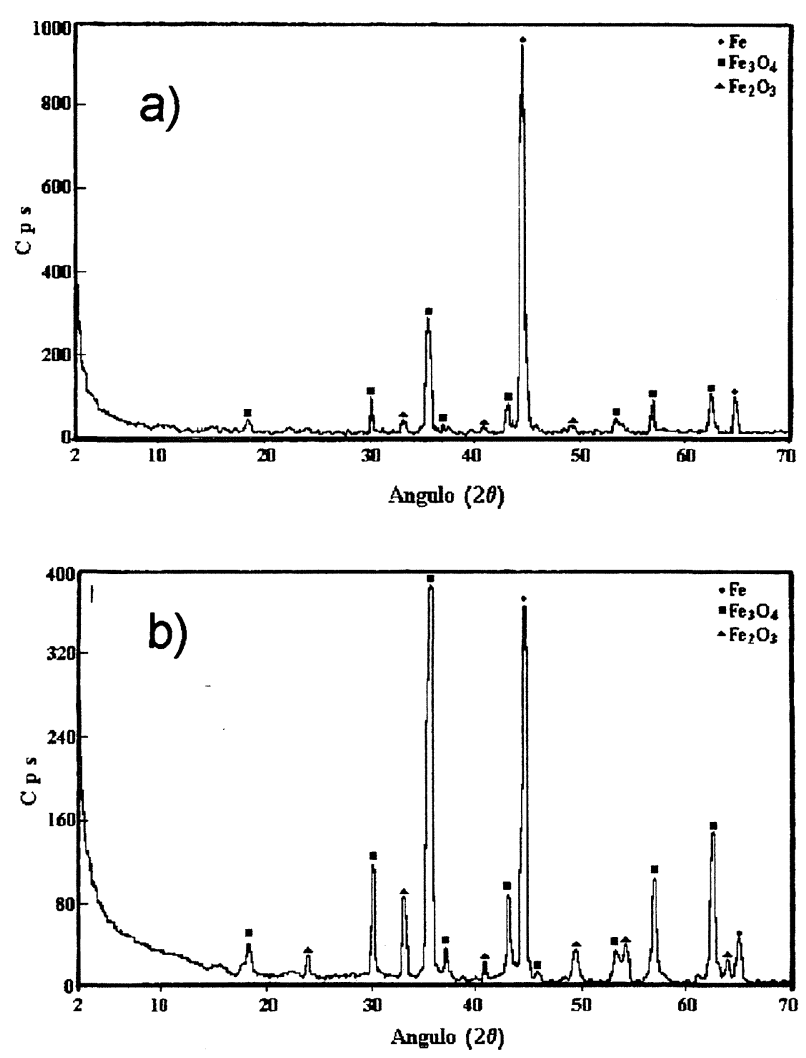

FIG. 11.- Difracción de rayos-X de los pelets reducidos a $900{ }^{\circ} \mathrm{C}$ y 25 min de carburización y reoxidadas a a) $300{ }^{\circ} \mathrm{C} \mathrm{y} \mathrm{b)} 400{ }^{\circ} \mathrm{C}$.

FIG. 11.-X-ray diffraction for $900{ }^{\circ} \mathrm{C}$ reduced pellets and 25 min carburization time and reoxidized at a) $300^{\circ} \mathrm{C}$ and b) $400^{\circ} \mathrm{C}$.

- Existe una clara tendencia de la reoxidación a disminuir cuando el proceso se efectúa a temperaturas de reducción de 900 y $1.000{ }^{\circ} \mathrm{C}$. Este efecto se presenta con mayor claridad a temperaturas de reoxidación de 250 y $300{ }^{\circ} \mathrm{C}$.

- El grado de reoxidación alcanzado disminuye cuando la temperatura de reducción aumenta.

- A temperaturas de reducción de 700 y $800{ }^{\circ} \mathrm{C}$, el tiempo de carburización no influye marcadamente en la reoxidación, pero a 900 y $1.000{ }^{\circ} \mathrm{C}$, se aprecia claramente un incremento en la reoxi- dación, es decir, a mayor tiempo de carburización mayor es la reoxidación.

- El grado de reoxidación es mayor a medida que la temperatura de reoxidación se incrementa.

- Los análisis de los pelets reoxidados revelan una reoxidación del tipo homogéneo especialmente en la superficie.

\section{Agradecimiento}

Los autores agradecen al CONACYT y a la Universidad michoacana de San nicolás de Hidalgo el apoyo brindado para la realización de este trabajo.

\section{REFERENCIAS}

1) Nixon, I.G. Ironmaking Steelmaking, 1, 1980: 2-12.

2) Jensen, H.B. y Smailer, R.M. Ironmaking Proc., 39, 1980: 430-436.

3) Iguchi, Y. e Inouye, M. Trans. ISIJ, 13, 1973: 274-282.

4) KaMiYa, K. y TANAKA, M: Trans. ISIJ, 21, 1981: 383-390.

5) Sraku-Lartey, K., Parker, R.H. y Hawkins, R.J. Ironmaking Steelmaking, 11 (1), 1984: 23-32.

6) Bandopadhyay, A., Ganguly, A., Prasad, K.K., SARKAR, S.B. y RAY, S.H. ISIJ Int., 29 (9), 1989: 753760.

7) Turkdogan, E.T. y Vinters, J.V. Metall. Trans., 5, 1974: 11-19.

8) Iguchi, Y. e Inouye, M. J. Iron Steel Inst. Japan, 56, 1970: 507-511.

9) El-Geassy, A.A., El-Kashif, F.O., NasR, M.I. y OMAR, A.A. ISIJ Int., 34 (7), 1994: 541-547.

10) Rao, M.N. y Lopez, J.R. Rev. Latinoam. Metal. Mater., 5 (1), 1985: 52-61.

11) Olsson, R.G. y Turkdogan, E.T, Metall. Trans., 5, 1974: 21-26.

12) El-Geassy, A.A., Shehata, K.A. y Ezz, S.Y. Trans. ISIJ, 17, 1977: 629-635.

13) Kaspersma, J.H. y Shay, R.H. Metall. Trans., 12B, (mar.) 1981: 77-82.

14) Towhidi, N. y Szekely, J. Metall. Trans., 14B (Sept.), 1983: 359-367.

15) Szekely, J. y Themelis, M.J. Rate Phenomena in Process Metallurgy, Ed. John Wiley. Nueva York, 1971: 601638. 